\title{
Philosophiques
}

\section{Richard Rorty, L'homme spéculaire (traduit de l'anglais par Thierry Marchaisse), Paris, Éditions du Seuil (coll. « L'ordre philosophique »), 1990, 439 pages.}

\section{Georges Leroux}

Volume 20, numéro 1, printemps 1993

URI : https://id.erudit.org/iderudit/027217ar

DOI : https://doi.org/10.7202/027217ar

Aller au sommaire du numéro

Éditeur(s)

Société de philosophie du Québec

ISSN

0316-2923 (imprimé)

1492-1391 (numérique)

Découvrir la revue

Citer cet article

Leroux, G. (1993). Richard Rorty, L'homme spéculaire (traduit de l'anglais par Thierry Marchaisse), Paris, Éditions du Seuil (coll. « L'ordre philosophique »), 1990, 439 pages. Philosophiques, 20(1), 209-211.

https://doi.org/10.7202/027217ar d'utilisation que vous pouvez consulter en ligne.

https://apropos.erudit.org/fr/usagers/politique-dutilisation/ 
Richard Rorty, L'homme spéculaire (traduit de l'anglais par Thierry Marchaisse), Paris, Éditions du Seuil (coll.

"L'ordre philosophique »), 1990, 439 pages.

\section{par Georges Leroux}

Quand il fit paraittre son livre Philosophy and the Mirror of Nature (Princeton University Press, 1980). Richard Rorty ne realisait sans doute pas à quel point 
il allait contribuer à réinstaller au cœur de la discussion épistémologique contemporaine une thématique relativiste qu'on croyait alors sur le déclin. La publication de la traduction française permet de mesurer le chemin qui a été parcouru au cours des dix dernières années. La pensée de Rorty a progressivement révélé sa grande complexité et le succès du livre de ig8o a entraîné un retour à l'ensemble de ses essais ${ }^{\mathrm{I}}$. Le portrait dualiste que plusieurs critiques avaient cherché à construire en opposant un empirisme épistémologique standard, assorti ou non de thèses historicistes, et un relativisme radical, repensé dans le cadre d'une théorie herméneutique de la culture, a dû être passablement nuancé. Au fur et à mesure que la discussion des thèses de l'ouvrage de I980 progressait, on s'aperçut de l'importance et de la féconditè des reformulations du pragmatisme proposées par Rorty. On dut aussi tenir compte des discussions élaborées que Rorty avait entreprises et développées avec la pensée continentale : pas seulement avec Michel Foucault, avec lequel il fut de bon ton de le confondre dans certains milieux de l'orthodoxie américaine, mais avec Jacques Derrida et avec Martin Heidegger, que de manière aussi originale que fructueuse il avait entrepris de relire dans le monde de pensée de John Dewey. Cette volonté de dialogue avec la pensée europeenne constitue certainement un des traits marquants de l'œuvre de Rorty et il ne s'agit pas d'un accident de surface : par le fond, elle est liée au désir de restituer à la philosophie une responsabilité de culture qui accepte l'héritage de la tradition, au lieu de toujours travailler à l'en débarrasser, et qui revendique une responsabilité d'interprétation, au lieu de chercher à en nier toujours davantage la pertinence ou la possibilité ${ }^{2}$. Il est donc question de penser pour la philosophie une fonction qui ne soit plus celle de la domination de tous les savoirs.

Une étude de la réception critique du livre permettrait de constituer une carte intéressante de l'epistémologie historique américaine. Pour une partie importante des philosophes qui reçurent ce livre avec interêt, il ètait grandement temps de compenser l'extrême spécialisation de la philosophie des sciences et de la connaissance par la réitération du projet herméneutique de la philosophie; que cette tâche doive enclencher une critique radicale de la tradition spéculative et que cette critique présente plusieurs embûches paraissait moins important que le programme d'une theorie enthousiaste de la culture. Mais pour d'autres, plus attentifs aux problèmes épistémologiques en tant que

1. Un seul recueil a été publié en français; il s'agit de Science et solidarité. La vérité sans le pouvoir. traduit par Jean-Pierre Cometti, Editions de l'Éclat (coll, « Tirè à part »), I990; ce recueil contient les quatre articles suivants : « Le pragmatisme, Davidson et la vérite » (rg86); « La science comme solidarité » ( 1987 ); « La science de la nature est-elle un genre naturel ? » (Ig88) et «Déconstruction et circonvention » (Ig84). Parmi les études publiées par Richard Rorty récemment, il faut mentionner Contingency, Iromy and Solidarity, Cambridge University Press, 1989, ainsi que deux volumes de Philosophical Papers, Cambridge University Press, I99I (volume I : Objectivity, Relativism and Truth et volume II : Essays on Heidegger and Others).

2. Cet aspect avait èté vu de manière très juste dans un des premiers comptes rendus publiès en français de Philosophy and the Mirror of Nature: il s'agit de la recension de Jacques Poulain, "Richard Rorty ou la boite blanche de la communication », Critique. numéro 417 (février I 982 ). 
tels, l'entreprise de Rorty apparaissait comme une déclaration généreuse, mais trop vague pour être prise au sérieux ${ }^{\mathrm{I}}$.

La traduction française permet de retourner à ce livre important avec un regard neuf. En accordant la place centrale à Heidegger, Wittgenstein et Dewey, Rorty indique dans quel paradigme il veut construire son entreprise : il s'agit. au-delà de Kant comme de toute pensée systématique, de « reconnaître que la notion de connaissance, en tant que représentation adéquate rendue possible par certains processus mentaux et rendue intelligible par une théorie générale de la représentation, doit être abandonnée. » L'ambition de l'ouvrage est thérapeutique, si on oppose ce terme au programme d'une construction de théories; elle valorise la proposition de pensées « édifiantes». Ce dernier terme acquiert le statut d'un concept important dans l'ouvrage, dans la mesure oủ la proposition de valeurs et de normes d'interprétation doit avoir préséance. selon Rorty, sur la représentation « spéculaire », c'est-à-dire sur la recherche illusoire d'un miroir exemplaire de la nature. La réflexion sur la connaissance doit donc en venir à accorder aux dimensions sociales et culturelles une importance qu'elles avaient perdue dans la conception abstraite qui dérive de Descartes.

L'ouvrage est divisé en trois parties : les deux premières produisent un travail à proprement parler déconstructeur des philosophies spéculatives de l'esprit, du mentalisme et du dualisme, ainsi que de leurs conséquences épistémologiques. Cette critique est menée sous l'égide d'une déconstruction des métaphores spéculaires ${ }^{2}$, mais on aurait tort de figer sous cette étiquette l'apport central de Rorty ; c'est plutôt le recours à un pragmatisme éclairé, seul susceptible de remplacer les théories de la représentation, qui constitue le thème le plus substantiel de cette partie, et en particulier du chapitre 4, dans lequel Rorty discute les idees de son maître Sellars. Pour une bonne part en effet, la première partie du livre enfonce des portes qui avaient été ouvertes par la philosophie analytique, et notamment par la critique de Gilbert Ryle du concept d'esprit. Le mérite de Rorty est de prendre au sérieux toutes les conséquences qui découlent de cette critique. C'est ici qu'intervient la déstabilisation du paradigme de l'objectivité des représentations privilégiées: si on suit Rorty à travers les quatre chapitres de la deuxième partie, on parvient à un point où il faut renoncer aux structures immuables produites par la philosophie en tant que théorie de la connaissance, et même à leurs substituts analytiques modernes comme la philosophie du langage (critiquée ici à travers l'entreprise de Davidson). En fait, Rorty montre surtout qu'aucune justification de la connaissance ne peut nous être fournie par le modèle spéculaire ou représentationnel, prèsenté ici dans sa version classique (et non dans quelque théorie cognitive récente de la représentation, présumément toutes conçues par lui comme des

I. Voir par exemple la réaction exacerbèe et à plusieurs égards très éclairante de Peter Muız. "Philosophy and the Mirror of Rorty », Philosophy of the Social Sciences (Ig84). L'auteur defend une position poppérienne.

2. On ne peut pas ne pas mentionner la proximité de cet aspect du travail de Rorty avec celui entrepris au mème moment par Robert Hébert sur les métaphores de la réflexion, dans son Mobiles du discours philosophique, Montréal. Hurtubise HMH, ig78. 
dérivés). Comme plusieurs critiques l'ont signalé, le besoin d'une justification lui semble incontournable, contrairement à ce qui se produit dans la théorie de Karl Popper. Cette exigence le porte à durcir l'alternative entre un justificationnisme rigide et un relativisme basé sur le consensus de la communauté. Quelle est la nature de l'autorité épistémique de cette communauté ? Pour répondre à cette question, il faut aller aux autres écrits de Rorty sur le pragmatisme.

La troisième partie est sans doute celle qui donne au livre sa tonalité inimitable : à la confrontation épistémologique issue du projet kantien, elle cherche à substituer un modèle « conversationnel », cest-à-dire une théorie fondée sur le relativisme de l'échange, de la communication et de la justification circonstancielle. Dans ce modèle, aucune discipline ne possède un privilège épistémologique. Dans un point de jonction inattendu, l'herméneutique européenne fait front commun avec le pragmatisme. Ces deux chapitres sont, dix ans après leur première publication, aussi stimulants et provocants. Si on accepte de mettre au rancart le programme spéculaire de la théorie de la connaissance, se voit-on d'emblée contraint à un relativisme violent ? Se met-on en position de devoir renoncer à toute forme de rationalité ? Rorty rejoint ici les intuitions de la pragmatique européenne en disposant la réponse sur le terrain du dialogue et d'un modèle dialogué de la culture ; la force motrice n'en est plus le désir de certitude, mais l'espoir d'édification. La dynamique profondément morale de ces deux chapitres contribue à faire basculer la pensée de l'épistèmé dans celle de la phronèsis. Il est donc question d'une autre rationalité, alimentée à des exigences de solidarité et consciente de son irréductible contingence. Son modèle est celui de l'herméneutique de Gadamer, utilisée ici comme méthode de la conversation infinie. Ces chapitres ont enclenché une discussion qui se poursuit dans plusieurs milieux de l'épistémologie contemporaine; ils ont également favorisé un rapprochement avec des disciplines comme la littérature et l'histoire qui avaient souffert de l'arrogance de « la représentation privilégiée » pendant trop longtemps. Qu'on s'accorde ou non avec le pragmatisme réinterprété de Richard Rorty, on ne peut que reconnaître la force de ses analyses et la vigueur de ses critiques : des questions comme celles de la nature de la communauté épistémique, la nécessité de la justification, la légitimité de l'herméneutique pour soutenir un relativisme mitigé ne trouvent pas ici toutes les réponses qu' on souhaiterait, mais elles se trouvent posées comme elles ne l'avaient pas été depuis très longtemps. La traduction française de Thierry Marchaisse est claire et élégante et elle montre une grande fidélité aux subtilités analytiques des discussions de Rorty; le lecteur français pourra s'y fier entièrement.

\section{Departement de philosophie}

Université du Québec à Montréal 